\title{
New Characterizations of the Pareto Distribution
}

\author{
Zohdy M. Nofal \\ Department of Statistics, Faculty of Commerce, Benha University, Egypt. \\ dr.zohdynofal@fcom.bu.edu.eg \\ Yehia M. El Gebaly \\ Department of Statistics, Faculty of Commerce, Benha University, Egypt. \\ yehia1958@yahoo.com
}

\begin{abstract}
Characterization results have great importance in statistics and probability applications. Some characterizations of Pareto of the first kind and Pareto of the second kind distributions are presented by using conditional expectation in terms of their failure (hazard) rate. We also provide two characterization theorems based on the $r$ th truncated moments.
\end{abstract}

Keywords: Characterization, Failure Rate, Conditional Expectation, Mixture, Pareto of The first kind, Pareto of The second kind Distributions.

\section{Introduction}

In recent years order statistics and their moments have assumed considerable interest, the moments of order statistics have been tabulated quite extensively for several distributions, for example see Arnold et al. (1992) and David (1981). Many papers dealing with characterization through properties of order statistics are appeared, see for example Khan and Abouammoh (1999), Malik et al. (1988), Lin (1988), Kamps ((1991), (1995)), and Mohie El-Din et al. (1991).

Khan and Abu-Salih (1989) have characterized many well-known continuous probability distributions such as Pareto and power function distributions through conditional expectation of functions of order statistics. Ahsanullah and Raqab (2004) have characterized continuous distributions by conditional expectation of some functions of generalized order statistics. Ahsanullah and Hamedani (2007) characterized beta of the first kind and the power function distribution using $1^{\text {st }}$ order statistics and $n^{\text {th }}$ order statistics respectively. Hamedani et al. (2008) characterized certain univariate distributions using truncated moments $X_{(1)}$. We like to mention here the works of Galambos and Kotz (1978), Kotz and Shanbag (1980), Ahsanullah (1989), Oncel et al. (2005) and Wesolowski and Ahsanullah (2004). Ahsanullah (2009) characterized several univariate distributions by the moments of the $(i+1)^{t h}(1 \leq i \geq n)$ order statistic given $i^{\text {th }}$ order statistic $=t$. In this paper characterizations of some univariate distributions using the $s^{\text {th }}$ moments of the $(r+1)^{t h}$ order statistic given $r^{\text {th }}$ order statistic $=x$ are given. Afify et al. (2013) characterized the exponential and power function distributions using the $s$ th conditional expectation of order statistics.

Let $X_{1}, X_{2}, \ldots, X_{n}$ be a random sample of size $n$ from an absolutely continuous distribution with cumulative distribution function $(c d f) F(x)$ and the corresponding probability density function $(p d f) f(x)$. Let $X_{(1)}, X_{(2)}, \ldots, X_{(n)}$ be the corresponding order 
statistics. Then the $p d f$ of $X_{(r)}$, the joint $p d f$ of $X_{(r)}$ and $X_{(r+1)}$ and the conditional $p d f$ of $X_{(r+1)}$ given $X_{(r)}=x$ are, respectively, see Arnold et al. (1992).

$$
\begin{gathered}
f_{X_{(r)}}(x)=\frac{n !}{(r-1) !(n-r) !} f(x)[F(x)]^{r-1}[1-F(x)]^{n-r}, a<x<b \\
f_{X_{(r)}, X_{(r+1)}}(x, y)=\frac{n !}{(r-1) !(n-r-1) !} f(x) f(y)[F(x)]^{r-1}[1-F(y)]^{n-r-1}, a<x<y<b \\
f_{X_{(r+1)} \mid X_{(r)}}(y \mid x)=(n-r) \frac{[1-F(y)]^{n-r-1}}{[1-F(x)]^{n-r}} f(y)
\end{gathered}
$$

In section 2, the Pareto of the first kind, Pareto of the second kind distributions are to be characterized through truncated moments of order statistics given by:

$$
\begin{gathered}
E\left(X_{(r+1)}^{s} \mid X_{(r)}=x\right)=\int_{x}^{\infty} y^{s} f_{X_{(r+1)} \mid X_{(r)}}(y \mid x) d y, \quad s=1,2,3, \ldots, \\
r=1,2, \ldots, n-1 .
\end{gathered}
$$

\section{Characterization Theorems}

\subsection{Characterization of Pareto of the First Kind Distribution}

The pdf and the survival function (sf) of the Pareto distribution of the first type are respectively,

$$
\begin{aligned}
& f(x)=\left(\frac{\beta}{\alpha}\right)\left(\frac{x}{\alpha}\right)^{-(\beta+1)}, \quad x \geq \alpha, \quad \beta>0, \quad \alpha>0 . \\
& \bar{F}(x)=\left(\frac{x}{\alpha}\right)^{-\beta}, \quad x \geq \alpha, \quad \beta>0, \quad \alpha>0 .
\end{aligned}
$$

\section{Theorem 2.1}

Let $X$ be a nonnegative continuous random variable with distribution function $F(\cdot)$, survival (reliability) function $\bar{F}(\cdot)$, density function $f(\cdot)$ and Failure (hazard) rate function $h(\cdot)$. Let $X_{(1)}, X_{(2)}, \cdots, X_{(n)}$ denote the order statistics of a random sample of size $n$ from $F(\cdot)$. The random variable $X$ has the Pareto distribution of the first type if and only if

$E\left(X_{(r+1)}^{s} \mid X_{(r)}=x\right)=x^{s}+\frac{s x^{s+1} h(x)}{\beta^{2}(n-r)-s \beta}, s=1,2, \ldots, r=1,2, \ldots, n-1, h(x)=\frac{\beta}{x}$.

\section{Proof.}

\section{(Necessity):}

Observe that

$$
E\left(X_{(r+1)}^{s} \mid X_{(r)}=x\right)=\int_{x}^{\infty} y^{s} f_{X_{(r+1)} \mid X_{(r)}}(y \mid x) d y .
$$


Using equation (1.3), we obtain

Where

$$
\begin{aligned}
E\left(X_{(r+1)}^{s} \mid X_{(r)}=x\right) & =\frac{n-r}{[1-F(x)]^{n-r}} \int_{x}^{\infty} \beta \alpha^{s-1}\left(\frac{y}{\alpha}\right)^{s-1}\left(\left(\frac{y}{\alpha}\right)^{-\beta}\right)^{n-r} d y \\
& =\frac{n-r}{[1-F(x)]^{n-r}} A .
\end{aligned}
$$

$$
\begin{aligned}
A & =\int_{x}^{\infty} \beta \alpha^{s-1}\left(\frac{y}{\alpha}\right)^{s-1}\left(\left(\frac{y}{\alpha}\right)^{-\beta}\right)^{n-r} d y \\
& =\frac{\beta x^{s}}{\beta(n-r)-s}\left(\left(\frac{x}{\alpha}\right)^{-\beta}\right)^{n-r}=\frac{\beta x^{s}}{\beta(n-r)-s}[1-F(x)]^{n-r} .
\end{aligned}
$$

Substitution equation (2.3) into equation (2.2), we obtain

$$
E\left(X_{(r+1)}^{s} \mid X_{(r)}=x\right)=x^{s}+\frac{s x^{s+1} h(x)}{\beta^{2}(n-r)-s \beta}
$$

\section{(Sufficiency):}

Notice that equation (2.4) can be reduced to

$$
\int_{x}^{\infty}(n-r) y^{s} f(y)[1-F(y)]^{n-r-1} d y=\left(x^{s}+\frac{s x^{s}}{\beta(n-r)-s}\right)[1-F(x)]^{n-r} \text {. }
$$

Differentiating the both sides of equation (2.5) with respect to $x$, we obtain

$$
\begin{aligned}
-(n-r) x^{s} f(x)(\bar{F}(x))^{n-r-1} & =-(n-r) x^{s} f(x)(\bar{F}(x))^{n-r-1} \\
& -\frac{(n-r) s x^{s}}{\beta(n-r)-s} f(x)(\bar{F}(x))^{n-r-1} \\
& +\left(\frac{\beta(n-r) s x^{s-1}}{\beta(n-r)-s}\right)(\bar{F}(x))^{n-r}, \\
\frac{(n-r) s x^{s} f(x)(\bar{F}(x))^{n-r-1}}{\beta(n-r)-s} & =\frac{\beta(n-r) s x^{s-1}(\bar{F}(x))^{n-r-1}}{\beta(n-r)-s} \\
x f(x) & =\beta \bar{F}(x),
\end{aligned}
$$

or equivalently

$$
\frac{f(x)}{\bar{F}(x)}=\frac{\beta}{x}
$$

Integrating the both sides of equation (2.6) with respect to $x$, we obtain

$$
\begin{aligned}
& \ln \bar{F}(x)=\ln x^{-\beta}+\ln k, \quad \text { where } k \text { is constant, } \\
& \ln \left(\frac{\bar{F}(x)}{k}\right)=\ln x^{-\beta}
\end{aligned}
$$




$$
\begin{gathered}
\frac{d}{d x} \bar{F}(x)=\frac{d}{d x} k x^{-\beta}, \\
f(x)=k \beta x^{-(\beta+1)}
\end{gathered}
$$

From the fact that $\int_{-\infty}^{\infty} f(x) d x=1$. Then $k=\alpha^{\beta}$, hence

$$
f(x)=\left(\frac{\beta}{\alpha}\right)\left(\frac{x}{\alpha}\right)^{-(\beta+1)}, \quad x \geq \alpha, \quad \beta>0, \quad \alpha>0 .
$$

Which is the $p d f$ of the Pareto distribution of the first type.

This completes the proof.

Remark 1. Specifying $s=1$ and $s=2$ in equation (2.1) yields the following results

(i) $E\left(X_{(r+1)} \mid X_{(r)}=x\right)=x+\frac{x}{\beta(n-r)-1}$.

(ii) $E\left(X_{(r+1)}^{2} \mid X_{(r)}=x\right)=x^{2}+\frac{2 x^{2}}{\beta(n-r)-2}$.

Then,

$$
\operatorname{Var}\left(X_{(r+1)} \mid X_{(r)}=x\right)=\frac{(n-r) x^{3} h(x)}{(\beta(n-r)-2)(\beta(n-r)-1)^{2}}
$$

Remark 2. Specifying s $=1$ in equation (2.1) gives the result of Ahsanullah (2009).

\subsection{Characterization of Pareto of the Second Kind Distribution}

In the sequel, we shall use the following symbol $m_{(r)}$

$$
m_{(r)}=m(m-1)(m-2) \ldots(m-r+1), \quad m \neq 0, r=1,2,3, \ldots .
$$

The pdf and the sf of the Pareto distribution of the second type are respectively,

$$
\begin{aligned}
& f(x)=\left(\frac{k}{\theta}\right)\left(1+\frac{x}{\theta}\right)^{-(k+1)}, x>0, k, \theta>0 . \\
& \bar{F}(x)=\left(1+\frac{x}{\theta}\right)^{-k}, x>0, k, \theta>0 .
\end{aligned}
$$

\section{Theorem 2.2}

Let $X$ be a nonnegative continuous random variable with distribution function $F(\cdot)$, survival (reliability) function $\bar{F}(\cdot)$, density function $f(\cdot)$ and Failure (hazard) rate function $h(\cdot)$. Let $X_{(1)}, X_{(2)}, \cdots, X_{(n)}$ denote the order statistics of a random sample of size $n$ from $F(\cdot)$. The random variable $X$ has the Pareto distribution of the second type if and only if

$$
\begin{aligned}
& E\left(X_{(r+1)}^{s} \mid X_{(r)}=x\right)=\sum_{j=0}^{s} \frac{m_{(1)} s ! x^{s-j} \theta^{j}\left(1+\frac{x}{\theta}\right)^{j}}{(s-j) ! m_{(j+1)}}, \quad m=k(n-r), \\
& r=1,2, \ldots, n-1, s=1,2,3, \ldots .
\end{aligned}
$$


The following two lemmas are used to prove the sufficiency of theorem 2.2. The two lemmas are proved in the appendix.

\section{Lemma 1}

$$
\frac{d}{d x} \sum_{j=0}^{s} \frac{m_{(1)} s ! x^{s-j} \theta^{j}\left(1+\frac{x}{\theta}\right)^{j}}{(s-j) ! m_{(j+1)}}=\frac{m_{(1)}}{\theta\left(1+\frac{x}{\theta}\right)} \sum_{j=1}^{s} \frac{m_{(1)} s ! x^{s-j} \theta^{j}\left(1+\frac{x}{\theta}\right)^{j}}{(s-j) ! m_{(j+1)}} .
$$

\section{Lemma 2}

$$
\sum_{j=0}^{s} \frac{m_{(1)} s ! x^{s-j} \theta^{j}\left(1+\frac{x}{\theta}\right)^{j}}{(s-j) ! m_{(j+1)}}=x^{s}+\sum_{j=1}^{s} \frac{m_{(1)} s ! x^{s-j} \theta^{j}\left(1+\frac{x}{\theta}\right)^{j}}{(s-j) ! m_{(j+1)}} .
$$

\section{Proof.}

\section{(Necessity):}

Observe that

$$
E\left(X_{(r+1)}^{s} \mid X_{(r)}=x\right)=\int_{x}^{\infty} y^{s} f_{X_{(r+1)} \mid X_{(r)}}(y \mid x) d y .
$$

Using equation (1.3), we obtain

$$
E\left(X_{(r+1)}^{s} \mid X_{(r)}=x\right)=\frac{n-r}{[1-F(x)]^{n-r}} \int_{x}^{\infty} \frac{k y^{s}}{y+\theta}\left[\left(1+\frac{y}{\theta}\right)^{-k}\right]^{n-r} d y
$$

Let $u=y+\theta$, then

$$
\begin{aligned}
E\left(X_{(r+1)}^{s} \mid X_{(r)}=x\right) & =\frac{n-r}{[1-F(x)]^{n-r}} \int_{x+\theta}^{\infty} \frac{k}{u}(u-\theta)^{s}\left(\frac{u}{\theta}\right)^{-k(n-r)-1} d u \\
& =\frac{n-r}{[1-F(x)]^{n-r}} A .
\end{aligned}
$$

Where

$$
A=\int_{x+\theta}^{\infty} \frac{k}{u}(u-\theta)^{s}\left(\frac{u}{\theta}\right)^{-k(n-r)-1} d u
$$

Integrating the right hand side of equation (2.9) by parts $s$ times, we obtain

$$
\begin{aligned}
A= & \frac{[1-F(x)]^{n-r}}{n-r}\left\{x^{s}+\frac{m_{(1) s ! x^{s-1} \theta\left(1+\frac{x}{\theta}\right)}}{(s-1) ! m_{(2)}}\right. \\
& \left.+\frac{m(1) s ! x^{s-2} \theta^{2}\left(1+\frac{x}{\theta}\right)^{2}}{(s-2) ! m_{(3)}}+\ldots+\frac{m_{(1) s ! \theta^{s}\left(1+\frac{x}{\theta}\right)^{s}}}{0 ! m_{(s+1)}}\right\} .
\end{aligned}
$$


Substituting equation (2.10) into equation (2.8), we obtain

$$
E\left(X_{(r+1)}^{s} \mid X_{(r)}=x\right)=\sum_{j=0}^{s} \frac{m_{(1)} s ! x^{s-j} \theta^{j}\left(1+\frac{x}{\theta}\right)^{j}}{(s-j) ! m_{(j+1)}}
$$

\section{(Sufficiency)}

Notice that equation (2.11) can be rewritten as follows:

$$
\begin{aligned}
& \int_{x}^{\infty}(n-r) y^{s} f(y)[1-F(y)]^{n-r-1} d y \\
& =[1-F(x)]^{n-r} \sum_{j=0}^{s} \frac{m_{(1)} s ! x^{s-j} \theta^{j}\left(1+\frac{x}{\theta}\right)^{j}}{(s-j) ! m_{(j+1)}} .
\end{aligned}
$$

Defferentiating the both sides of equation (2.12) with respect to $x$, we obtain

$$
\begin{aligned}
-(n-r) x^{s} f(x)(\bar{F}(x))^{n-r-1} & \\
& =(\bar{F}(x))^{n-r} \frac{d}{d x} \sum_{j=0}^{s} \frac{m_{(1)} s ! x^{s-j} \theta^{j}\left(1+\frac{x}{\theta}\right)^{j}}{(s-j) ! m_{(j+1)}} \\
& -(n-r) f(x)(\bar{F}(x))^{n-r-1} \sum_{j=0}^{s} \frac{m_{(1)} s ! x^{s-j} \theta^{j}\left(1+\frac{x}{\theta}\right)^{j}}{(s-j) ! m_{(j+1)}} .
\end{aligned}
$$

Using Lemma (1) and Lemma (2) and simplifying, we obtain

$$
\begin{aligned}
(n-r) f(x) \sum_{j=1}^{s} \frac{m_{(1)} s ! x^{s-j} \theta^{j}\left(1+\frac{x}{\theta}\right)^{j}}{(s-j) ! m_{(j+1)}} & =\frac{m_{(1)}}{\theta\left(1+\frac{x}{\theta}\right)} \bar{F}(x) \sum_{j=1}^{s} \frac{m_{(1)} s ! x^{s-j} \theta^{j}\left(1+\frac{x}{\theta}\right)^{j}}{(s-j) ! m_{(j+1)}}, \\
(n-r) f(x) & =\frac{m_{(1)}}{\theta\left(1+\frac{x}{\theta}\right)} \bar{F}(x),
\end{aligned}
$$

or equivalently

$$
\begin{aligned}
& \frac{f(x)}{\bar{F}(x)}=\frac{m_{(1)}}{\theta(n-r)\left(1+\frac{x}{\theta}\right)}, \text { where } m_{(1)}=k(n-r), \text { hence } \\
& \frac{f(x)}{\bar{F}(x)}=\frac{k}{\theta\left(1+\frac{x}{\theta}\right)} .
\end{aligned}
$$

Integrating the right hand side of equation (2.13) with respect to $x$, we obtain

$$
\int \frac{f(x)}{\bar{F}(x)} d x=\int \frac{k}{\theta\left(1+\frac{x}{\theta}\right)} d x
$$




$$
\begin{aligned}
& \ln \bar{F}(x)=-k \ln \left(1+\frac{x}{\theta}\right)+\ln c, \text { where } c \text { is constant } \\
& \ln \left(\frac{\bar{F}(x)}{c}\right)=\ln \left(1+\frac{x}{\theta}\right)^{-k} \\
& \bar{F}(x)=c\left(1+\frac{x}{\theta}\right)^{-k} .
\end{aligned}
$$

Using the fact that $\bar{F}(0)=1$. Then $c=1$.

Hence

$$
\bar{F}(x)=\left(1+\frac{x}{\theta}\right)^{-k}, x \geq 0, k, \theta>0
$$

Which is the $s f$ of the Pareto distribution of the second type.

This completes the proof.

Remark 3. Specifying $s=1$ and $s=2$ in equation (2.7) yields the following results

(i) $E\left(X_{(r+1)} \mid X_{(r)}=x\right)=x+\frac{x+\theta}{m-1}$.

(ii) $E\left(X_{(r+1)}^{2} \mid X_{(r)}=x\right)=x^{2}+\frac{2 x(x+\theta)}{m-1}+\frac{2(x+\theta)^{2}}{(m-1)(m-2)}$.

Then,

$$
\operatorname{Var}\left(X_{(r+1)} \mid X_{(r)}=x\right)=\frac{2 x(x+\theta)}{m-1}+\frac{m(x+\theta)^{2}}{(m-1)^{2}(m-2)} .
$$

\subsection{Characterization of Two Mixture of Pareto Distributions}

\section{Theorem 2.3}

Let $X$ be a nonnegative continuous random variable with distribution function $F(\cdot)$, survival (reliability) function density function $\bar{F}(\cdot)$, density function $f(\cdot)$, Failure (hazard) rate function $h(\cdot), E\left(X^{j}\right)=\mu^{j}$. The random variable $X$ has the Pareto distribution as

$$
f(x)=\beta \alpha^{\beta} x^{-\beta-1}, \quad \bar{F}(x)=\alpha^{\beta} x^{-\beta}, \quad x \geq \alpha, \alpha, \beta>0 .
$$

If and only if

$$
E\left(X^{j} \mid X \geq x\right)=\mu^{j} \frac{x^{j+1}}{\beta \alpha^{j}} h(x), \quad j<\beta .
$$

proof.

(Necessity):

Let $X$ has a Pareto distribution with $\alpha, \beta>0$ which is shap and scale parameters. Then

$$
\bar{F}(x) E\left(X^{j} \mid X \geq x\right)=\int_{x}^{\infty} u^{j} f(u) d u=\int_{x}^{\infty} \alpha^{\beta} \beta u^{j-\beta-1} d u
$$




$$
\begin{aligned}
& =\left.\alpha^{\beta} \beta \frac{u^{j-\beta}}{j-\beta}\right|_{x} ^{\infty}=-\frac{\beta \alpha^{\beta}}{j-\beta} x^{j-\beta} \\
& =\frac{x}{\beta-j} f(x)
\end{aligned}
$$

Then

$$
E\left(X^{j} \mid X \geq x\right)=\mu^{j} \frac{x^{j+1}}{\beta \alpha^{j}} h(x) .
$$

The proof is completed.

\section{(Sufficiency):}

Notice that equation (2.14) can be rewritten as

$$
\int_{x}^{\infty} u^{j} f(u) d u=\frac{x^{j+1}}{\beta-j} f(x) .
$$

By differentiating both sides with respect to $x$, then

$$
-x^{j} f(x)=\frac{x^{j+1}}{\beta-j} f^{\prime}(x)+\frac{(j+1) x^{j}}{\beta-j} f(x) .
$$

By gathering the simillar terms, then

$$
-x^{j+1} f^{\prime}(x)=(\beta+1) x^{j} f(x),
$$

or

$$
\frac{f^{\prime}(x)}{f(x)}=-\frac{(\beta+1)}{x^{j}}
$$

Integrating both sides, with respect to $x$, we get

$$
f(x)=k x^{-\beta-1} \text {. }
$$

Using the fact that $\int_{\alpha}^{\infty} f(x) d x=1$. Then $k=\beta \alpha^{\beta}$. Thus, we have

$$
f(x)=\beta \alpha^{\beta} x^{-\beta-1} \text {. }
$$

Which is the density function of the Pareto distribution.

Corollary 4. If $j=1$ then the first truncated moment will be as

$$
E(X \mid X \geq x)=\frac{x^{2}}{\beta-1} h(x) .
$$

Corollary 5. The mean residual life can be written as

$$
E((X-\mu) \mid X \geq x)=\frac{x^{2}}{\beta-1}[h(x)-\alpha \beta] .
$$


Corollary 6. if $j=2$ then the second truncated moment will be as

$$
E\left(X^{2} \mid X \geq x\right)=\frac{x^{3}}{\beta-2} h(x) .
$$

Corollary 7. Using corollary (4) and (6), we can compute the truncated variance as

$$
V(X \mid X \geq x)=\frac{x^{3}}{\beta-2} h(x)-\frac{x^{2}}{[\beta-1]^{2}}[h(x)]^{2} .
$$

\section{Theorem 2.4}

Let $X$ be a nonnegative continuous random variable with distribution function $F(\cdot)$, survival (reliability) function density function $\bar{F}(\cdot)$, density function $f(\cdot)$, reversed Failure (hazard) rate function $\eta(\cdot)$. Then $X$ has the Pareto distribution as

$$
f(x)=\beta \alpha^{\beta} x^{-\beta-1}, \quad \bar{F}(x)=\alpha^{\beta} x^{-\beta}, \quad x \geq \alpha, \quad \alpha>0, \quad \beta>0,
$$

if and only if

\section{Proof.}

$$
E\left(X^{j} \mid X \leq x\right)=\mu^{j}+\frac{x}{\beta-j}\left(\alpha^{j}-x^{j}\right) \eta(x) .
$$

\section{(Necessity):}

Let $X$ has a Pareto distribution with parameters $\alpha>0, \beta>0$. Then,

$$
\begin{aligned}
F(x) E\left(X^{j} \mid X \leq x\right) & =\int_{\alpha}^{x} u^{j} f(u) d u=\int_{\alpha}^{x} \alpha^{\beta} \beta u^{j-\beta-1} d u \\
& =\left(\left.\alpha^{\beta} \beta \frac{u^{j-\beta}}{j-\beta}\right|_{\alpha} ^{x}\right)=\frac{\beta \alpha^{\beta}}{j-\beta}\left[x^{j-\beta}-\alpha^{j-\beta}\right] .
\end{aligned}
$$

After adding some terms and rearranging the terms, we get

$$
F(x) E\left(X^{j} \mid X \leq x\right)=\mu^{j} F(x)+\frac{x}{\beta-j} f(x)\left[\alpha^{j}-x^{j}\right] .
$$

Then

$$
E\left(X^{j} \mid X \leq x\right)=\mu^{j}+\frac{x}{\beta-j} \eta(x)\left[\alpha^{j}-x^{j}\right]
$$

The proof is completed.

\section{(Sufficiency):}

Notice that the last equation can be rewritten as

$$
(\beta-j) \int_{\alpha}^{x} u^{j} f(u) d u=\beta \alpha^{j} F(x)+x f(x)\left[\alpha^{j}-x^{j}\right] .
$$

By differentiating the both sides with respect to $x$ then,

$$
(\beta-j) x^{j} f(x)=\beta \alpha^{j} f(x)+x f^{\prime}(x)\left[\alpha^{j}-x^{j}\right]+f(x)\left[\alpha^{j}-(j+1) x^{j}\right] .
$$


By gathering the simillar terms, then

$$
-x f^{\prime}(x)=-(\beta+1) f(x)
$$

or

$$
\frac{f^{\prime}(x)}{f(x)}=-\frac{(\beta+1)}{x^{j}}
$$

Integrating the both sides, with respect to $x$, we get $f(x)=k x^{-\beta-1}$. Using the fact that

$$
\int_{\alpha}^{\infty} f(x) d x=1
$$

Then $k=\beta \alpha^{\beta}$ and $f(x)=\beta \alpha^{\beta} x^{-\beta-1}$ which is the density function of the Pareto distribution.

Corollary 8. If $j=1$, then the first truncated moment will be as

$$
E(X \mid X \leq x)=\mu+\frac{x}{\beta-1}(\alpha-x) \eta(x) \text {. }
$$

Corollary 9. The mean inactivity time can be written as

$$
E((\mu-X) \mid X \geq x)=-\frac{x}{\beta-1}(\alpha-x) \eta(x) \text {. }
$$

Corollary 10. If $j=2$, then the second truncated moment will be as

$$
E\left(X^{2} \mid X \leq x\right)=\mu^{2}+\frac{x}{\beta-2} \eta(x)\left[\alpha^{2}-x^{2}\right]
$$

Corollary 11. Using corollary (8) and (10), we can compute the truncated variance as

$$
V(X \mid X \leq x)=\frac{x(\alpha-x) \eta(x)}{(\beta-2)(\beta-1)^{2}}\left[(\alpha+x)(\beta-1)^{2}-2 \alpha \beta(\beta-2)\right]-\frac{x^{2}(\alpha-x)^{2} \eta^{2}(x)}{(\beta-1)^{2}}
$$

\section{Acknowledgements}

The authors would like to thank the Editor in Chief and the referees for very careful reading and valuable comments which greatly improved the paper.

\section{References}

1. Afify, A. Nofal, Z. M. Ahmed, A. N. (2013). Characterization of exponential and power function distributions Using sth truncated moments of order statistics. Journal of Advances in Mathematics, 4, 486-495.

2. Ahsanullah, M. (2009). On some characterizations of univariate distributions based on truncated moments of order statistics. Pak. J. statist. 25, 83-91.

3. Ahsanullah, M. (1989). On characterizations of the uniform distribution based on functions of order statistics. Aligarh J. Statistics, 9, 106. 
4. Ahsanullah, M. and Hamedani, G.G. (2007). Certain characterizations of power function and Beta distributions based on order statistics. J. Statist.Theor. Appl., 6, 220-226.

5. Ahsanullah, M. and Raqab M. Z. (2004) Characterizations of distributions by conditional expectations of generalized order statistics. J. Appl. Statist. Sc., 13, 41-48.

6. Arnold, B.C., Balakrishnan, N. and Nagaraja, H. N. (1992). A First Course in Order Statistics, John Wiley \& Sons, New York.

7. David, H .A. (1981) Order statistics, 2nd Ed. John Wiley \& Sons, New York.

8. Galambos, J. and Kotz, S. (1978). Characterizations of probability distri- -butions. A unified approach with an emphasis on exponential and related models. Lecture Notes in Mathematics, 675, Springer Verlag.

9. Hamedani, G.G., Ahsanullah, M. and Sheng, R. (2008). Characterizations of certain continuous univariate distributions based on truncated moment of the first order statistics, Aligarh J Stat., 28, 75-81.

10. Kamps, U. (1991). A general recurrence relation for moments of order statistics in a class of probability distributions and characterizations. Metrika, 38, 215-225.

11. Kamps, U. (1995). A concept of generalized order statistics. Teubner, Stuttgart.

12. Khan, A. H. and Abouammoh, A. M. (1999). Characterizations of distributions by conditional expectation of order statistics, 9, 159-168.

13. Khan, A.H. and Abu-Salih MS (1989). Characterizations of probability distributions by conditional expectation of order statistics. Metron, 47, 171-181.

14. Kotz, S. and Shanbhag, D.N. (1980). Some new approaches to probability distributions. Adv. in Appl. Probab., 12, 903-912.

15. Lin, G. D. (1988). Characterizations of distributions via relationships between two moments of order statistics. J. Statist.Plan. Inf., 19, 73-80.

16. Malik, H. J., Balakrishnan, N. and Ahmed, S. E. (1988). Recurrence relations and identities for moments of order statistics, I: Arbitrary continuous distributions. Commun. Statist. Theor. Meth., 17, 2623-2655.

17. Mohie El-Din, M.M., Mahmoud, M.A.W. and Abu-Youssef, S.E. (1991). Moments of order statistics from parabolic and skewed distributions and characterization of Weilbull distribution. Commun. Statist. Simul.Comput., 20, 639-645.

18. Oncel, S.Y., Ahsanullah, M., Aliev, F.A. and Aygun, F. (2005). Switching record and order statistics via random contraction. Statist. Probab. Lett., 73, 207-217.

19. Wesolowski, J. and Ahsanullah, M. (2004). Switching order statistics through random power contraction. Aust. N.Z.J. Statist., 6, 297-303. 


\section{Appendix}

\section{Proof of Lemma 1}

$$
\begin{aligned}
& \frac{d}{d x} \sum_{j=0}^{s} \frac{m_{(1)} s ! x^{s-j} \theta^{j}\left(1+\frac{x}{\theta}\right)^{j}}{(s-j) ! m_{(j+1)}}= \\
& {\left[s x^{s-1}+\frac{m_{(1) s ! x^{s-1}}}{(s-1) ! m_{(2)}}\right]+\left[\frac{m_{(1) s ! x^{s-2} \theta\left(1+\frac{x}{\theta}\right)}}{(s-2) ! m_{(2)}}+\frac{2 m_{(1) s ! x^{s-2} \theta\left(1+\frac{x}{\theta}\right)^{s-1}}}{(s-3) ! m_{(3)}}\right]} \\
& +\ldots+\left[\frac{m_{(1) s ! \theta^{s-1}\left(1+\frac{x}{\theta}\right)}}{0 ! m_{(s)}}+\frac{s m_{(1) s ! \theta^{s-1}\left(1+\frac{x}{\theta}\right)^{s-1}}}{0 ! m_{(s+1)}}\right] \\
& =m_{(1)} \frac{m_{(1) s ! x^{s-1}}}{(s-1) ! m_{(2)}}+m(1) \frac{m_{(1) s ! x^{s-2} \theta\left(1+\frac{x}{\theta}\right)}}{(s-2) ! m_{(3)}} \\
& +\ldots+m_{(1)} \frac{m_{(1) s ! \theta^{s-1}\left(1+\frac{x}{\theta}\right)^{s-1}}}{0 ! m_{(s+1)}} \\
& =\frac{m_{(1)}}{\theta\left(1+\frac{x}{\theta}\right)}\left[\frac{m_{(1) s ! x^{s-1}} \theta\left(1+\frac{x}{\theta}\right)}{(s-1) ! m_{(2)}}+\frac{m_{(1) s ! x^{s-2} \theta^{2}\left(1+\frac{x}{\theta}\right)^{2}}}{(s-2) ! m_{(3)}}\right. \\
& \left.+\ldots+\frac{m_{(1) s ! \theta^{s}\left(1+\frac{x}{\theta}\right)^{s}}}{0 ! m_{(s+1)}}\right] \\
& =\frac{m_{(1)}}{\theta\left(1+\frac{x}{\theta}\right)} \sum_{j=1}^{s} \frac{m_{(1)} s ! x^{s-j} \theta^{j}\left(1+\frac{x}{\theta}\right)^{j}}{(s-j) ! m_{(j+1)}} \text {. }
\end{aligned}
$$

\section{Proof of Lemma 2}

$$
\begin{aligned}
& \sum_{j=0}^{s} \frac{m_{(1)} s ! x^{s-j} \theta^{j}\left(1+\frac{x}{\theta}\right)^{j}}{(s-j) ! m_{(j+1)}}=x^{s}+\left[\frac{m_{(1) s ! x^{s-1}} \theta\left(1+\frac{x}{\theta}\right)}{(s-1) ! m_{(2)}}+\frac{m_{(1) s ! x^{s-2} \theta^{2}\left(1+\frac{x}{\theta}\right)^{2}}}{(s-2) ! m_{(3)}}\right. \\
& \left.\quad+\cdots+\frac{m_{(1) s !} \theta^{s}\left(1+\frac{x}{\theta}\right)^{s}}{0 ! m_{(s+1)}}\right] \\
& =x^{s}+\sum_{j=1}^{s} \frac{m_{(1)} s ! x^{s-j} \theta^{j}\left(1+\frac{x}{\theta}\right)^{j}}{(s-j) ! m_{(j+1)}} .
\end{aligned}
$$

